\title{
Monitoring de turbine hydraulique
}

\author{
Dimitry Khaletzky \\ Société Neyrpic-GEC Alsthom \\ Grenoble
}

\section{Introduction}

Un groupe hydraulique est surveillé classiquement par des dispositifs électriques chargés de fermer un contact lorsqu'une grandeur mesurée dépasse un seuil prédéfini. Les appareils les plus usuels sont les pressostats, thermostats et seuils de niveau. On peut citer également les seuils de vitesse et les seuils de niveau vibratoire. Avec cette technologie, le réglage des seuils est effectué a priori et figé pour l'exploitation. Certains réglages tels que niveau haut, niveau bas, pression, survitesse, ne présentent pas de difficulté. Les températures et niveaux vibratoires acceptables peuvent au contraire varier d'une machine à l'autre, ne sont pas connus exactement lors des études et peuvent être dépendants de conditions extérieures. Par suite, les seuils sur ces variables sont généralement calés assez haut, afin d'éviter les alarmes intempestives. Il s'en suit que certaines anomalies sont détectées lors d'une panne confirmée, alors qu'une surveillance plus fine aurait pu générer un diagnostic plus précoce et plus précis.

Pour mieux utiliser les mesures continues disponibles sur un groupe hydraulique, il faut :

- déterminer leur évolution normale en fonction de paramètres extérieurs tels que la puissance produite ou la température extérieure ;

- prendre en compte ces variations de niveau admissible durant la surveillance en temps réel de la machine.

\section{Monitoring vibratoire}

De nombreux groupes turboalternateurs dans le monde entier, sont aujourd'hui équipés de capteurs de vibrations. Les capteurs sont du type relatif (capteurs de déplacement à courants de Foucault) ou absolus (accéléromètres audessus de $3 \mathrm{Hertz}$, vélocimètres compensés entre 1 et 3 Hertz). Le critère de choix entre les deux mesures est essentiellement la rigidité du palier. Sur une petite turbine Pelton horizontale, on installe typiquement deux capteurs de déplacement $\mathrm{XY}$ et un capteur de référence de phase. Sur une turbine Francis verticale de forte puissance le palier vibre avec l'arbre, si bien qu'une mesure absolue n'aurait pas de sens. Dans ce cas, l'on choisit des accéléromètres ou vélocimètres, suivant la vitesse de rotation.

Ces chaînes de mesures vibratoires fournissent généralement un signal continu représentatif du niveau de vibration : valeur efficace, crête-crête, etc... Ce signal est susceptible de déclencher un ou plusieurs seuils électriques. Le signal du capteur lui-même est également disponible. Un opérateur muni d'un matériel portable, peut à intervalles réguliers effectuer des analyses de Fourier, ou tracer des orbites dans le cas des capteurs de déplacement. Une telle surveillance périodique humaine peut mettre en évidence une évolution anormale de la machine.

Cette stratégie de surveillance conduit à deux conclusions importantes :

\section{Hydraulic turbine monitoring}

Early detection of a unit failure greatly increases its reliability. To do this, an accurate knowledge of the unit behaviour is required. Obviously, computer aided data storage and analysis will increase this knowledge. However, its success depends on the good choice of sensors and an efficient method of data analysis. We propose a concrete approach to set up progressively an accurate and efficient remote unit supervision. 
- les mesures vibratoires n'ont une signification qu'en comparaison avec un état de référence,

- il existe deux façons d'utiliser les mesures d'un capteur : un mode élémentaire en ligne consistant à comparer une mesure continue à une référence, et un travail plus complexe exécuté hors ligne par un spécialiste, destiné à mieux connaître la machine afin de détecter les anomalies d'une façon beaucoup plus fine et donc plus précoce.

\section{Traitement informatique des mesures}

Les conclusions ci-dessus s'appliquent en fait à toutes les mesures analogiques: d'une part les mesures déclenchent des alarmes lorsqu'elles sortent des plages de fonctionnement normal, d'autre part l'analyse humaine de ces mesures permet de mieux connaître la machine et de définir précisément les plages de fonctionnement pour chaque variable.

Il est clair qu'un stockage informatique des enregistrements représente une aide précieuse, car il en permet un traitement différé avec des logiciels de dépouillement performants. Toutefois, on se rend compte également que des plages précises doivent nécessairement dépendre des conditions de fonctionnement. Cette adaptation de plage doit être réalisée par un calculateur temps réel.

L'organisation du système de surveillance prend alors la forme indiquée sur le schéma ci-dessous:

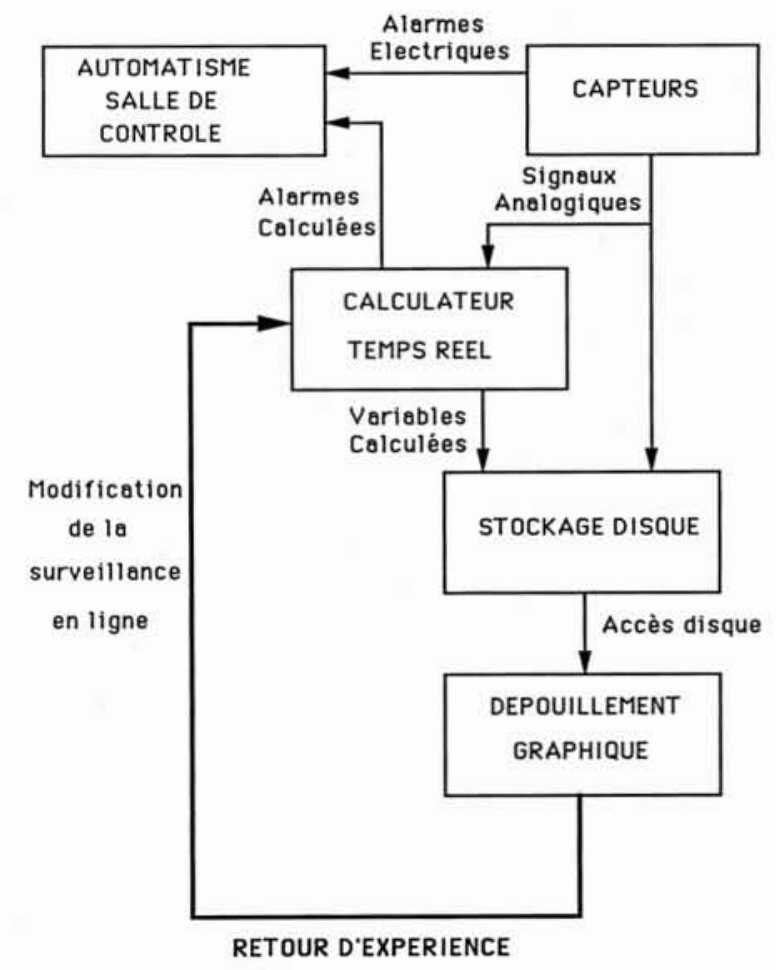

LA HOUILLE BLANCHE/N²-1991

\section{Stockage et dépouillement des mesures}

Le stockage disque de données analogiques nécessite un compromis entre la fréquence de stockage et l'espace disque disponible. La solution généralement retenue est de ne stocker que les régimes transitoires. Une turbine hydraulique peut supporter une trentaine de capteurs analogiques de tous types (vibrations, pressions, températures, positions, vitesses, puissances, niveaux). Un enregistrement de 30 mesures simultanées occupe donc 60 octets. Une disquette peut contenir 20000 enregistrements, alors qu'un disque dur de $20 \mathrm{Mo}$ en contiendra plus de 330000 . Un tel disque peut suffire pour enregistrer durant une année, avec une période d'une seconde, des transitoires quotidiens de 30 minutes.

Cette base de données permet:

— une analyse différée des incidents ;

- une aide au bilan d'exploitation (énergie produite, débit turbiné, rendement global, durée de fonctionnement en zone déconseillée (cavitation)) ;

- une définition plus précise des plages de fonctionnement normales de chaque variable surveillée;

- la découverte de nouvelles relations entre variables, permettant de mieux connaître la machine et d'affiner la surveillance en ligne. On sait par exemple que la température palier est aussi fonction de la température de l'eau. On pourra également rechercher une relation entre le niveau de vibration admissible et la puissance.

\section{Traitement des mesures en temps réel}

Nous avons vu que le calculateur temps réel a pour rôle principal de comparer en permanence les mesure à des valeurs tabulées telles que:

$$
\text { Tmaxi palier }=\mathrm{f}(\mathrm{W} \text { elec, } \mathrm{T} \text { huile, } \text { Tair })
$$

Il peut également être utile de filtrer certaines mesures (passe-bas, passe-haut, passe-bande), de réaliser des combinaisons logiques (alarme

si.. et ... ou non ....) et des calculs arithmétiques $(+,-, *, /$, racine carrée, valeur absolue...)

Il faut enfin que toutes ces fonctions soient programmables par l'utilisateur sans modification du logiciel temps réel.

\section{Description du matériel}

Les capteurs et leurs modules de conditionnement sont fixés sur la machine. Les signaux entrent dans une armoire électrique, via des modules électroniques spécifiques à chaque capteur.

Les signaux vibratoires sont traités par des modules analogiques qui déterminent la valeur continue nécessaire (Valeur efficace, crête, valeur absolue, Smax pour les déplacements X-Y...) et actionnent des contacts électriques. 
Les signaux entrent ensuite dans un rack digital compatible IBM-PC, qui contient au minimum :

- une carte à microprocesseur exploitant le logiciel de fonctions d'automatismes LARRY (marque déposée par Neyrpic);

- des sorties série, clavier, écran et imprimante ;

- des cartes de sorties logiques afin d'envoyer les alarmes calculées vers l'automatisme, ou la salle de contrôle ;

— un disque dur ;

- un lecteur de disquette.

La disquette contient les enregistrements les plus récents, alors que le disque dur doit stocker toutes les informations concernant une machine pendant un an. Les dépouillements périodiques se font en connectant temporairement les périphériques nécessaires (écran, clavier, imprimante, traceur..) au rack digital.

Le matériel du commerce permet toutes sortes d'extensions à cette configuration minimale. Citons par exemple : - écran, clavier et imprimante à demeure dans l'armoire ; - liaison modem permettant le transfert des données acquises vers le bureau d'études ;

- carte de communication avec un réseau d'automates; - centralisation des données sur un calculateur maître par réseau de fibres optiques.

\section{Conclusions}

La Société Neyrpic possède une très longue expérience dans la construction des turbines hydrauliques et leur régulation, qui lui permet de définir une instrumentation adaptée et d'effectuer une analyse efficace des mesures, afin d'améliorer la télésurveillance d'un groupe.

Le département Régulations et Automatismes possède une maitrise des matériels et logiciels d'informatique temps réel, qui lui permet :

- de mettre en place un système de surveillance adapté au besoin de l'exploitation;

— de le connecter à tout système informatique de contrôle ;

- d'effectuer l'analyse périodique des données collectées afin d'affiner progressivement la surveillance en ligne.

Cet équipement réalise une surveillance en ligne très adaptée à la machine et évolutive, ce qui permet d'anticiper largement sur un accident majeur, tel qu'une fissuration d'arbre. 